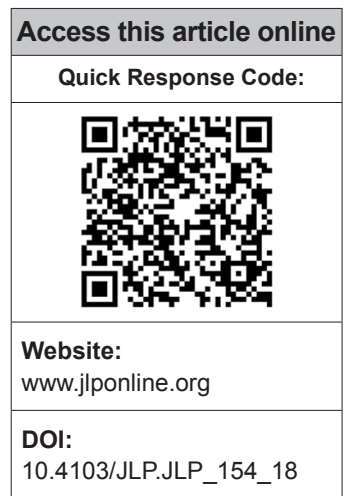

Department of Microbiology, All India Institute of Medical Sciences, Raipur, Chhattisgarh, India

Address for correspondence: Dr. Padma Das,

Department of

Microbiology, All India Institute of Medical Sciences, Raipur Gate No 5, Medical College Building, First Floor Tatibandh, GE Road, Raipur, Chhattisgarh, India. E-mail: drdaspadma@ gmail.com

Submission: $23-11-2018$ Accepted: 31-05-2019

\title{
Estimating the burden of enteric fever in Chhattisgarh: A single-center study on culture-positive cases from a newly built tertiary care hospital
}

\author{
Debabrata Dash, Padma Das, Anudita Bhargava, Ujjwala Nitin Gaikwad, \\ Sanjay Singh Negi, Archana Wankhede
}

\section{Abstract:}

BACKGROUND: Enteric fever is the most common cause of community acquired blood stream infections in under developed and developing countries. The enteric fever is exclusive to humans and transmitted through the faeco-oral route. Though India is an endemic zone for enteric fever, the data is very scarce from Central India. The present study was undertaken to determine the prevalence of enteric fever in this region and to know the antimicrobial susceptibility pattern of the isolated typhoidal Salmonellae.

MATERIAL AND METHOD: We conducted a retrospective analysis of blood culture positive cases of enteric fever over a period of two years (December 2015 to December 2017). All blood cultures submitted for suspected enteric fever and associated symptoms were included in the study. Relevant demographic, clinical and laboratory data were analyzed.

RESULT: A total of 51 cases (3.56\%) were of typhoidal Salmonella from a total of 1430 blood culture submission. Salmonella Typhi were $70.5 \%$ while Salmonella Paratyphi A were $29.5 \%$ of the total isolated Salmonellae. The most vulnerable age group was $10-19 y e a r s ~(41.2 \%)$. The mean minimum inhibitory concentration of ciprofloxacin for Salmonella Typhi and Salmonella Paratyphi A are 1.20 and $1.97 \mu \mathrm{g} / \mathrm{ml}$ respectively. All the isolates were susceptible to ceftriaxone. Highest isolation was in the July - September quarter (35.3\%).

CONCLUSION: There is a high prevalence of the disease which needs urgent focus on safe water, sanitation services and also to establish guidelines for empiric therapy for enteric fever.

Key words:

Bloodstream infection, ciprofloxacin resistance, enteric fever, Salmonella

\section{Introduction}

Salmonella enterica serovar Typhi, $\checkmark$ Paratyphi A, Paratyphi B, and Paratyphi C collectively known as typhoidal Salmonella is the most common cause of community-acquired bloodstream infections in underdeveloped and developing countries ${ }^{[1]}$ Typhoid fever and paratyphoid fever as they are known to be; depending

This is an open access journal, and articles are distributed under the terms of the Creative Commons Attribution-NonCommercial-ShareAlike 4.0 License, which allows others to remix, tweak, and build upon the work non-commercially, as long as appropriate credit is given and the new creations are licensed under the identical terms.

For reprints contact: reprints@medknow.com on whether the infection is caused by $S$. enterica serovar Typhi or any one of the $S$. enterica serovar Paratyphi A, Paratyphi B, or Paratyphi $C$, respectively, are together referred to as enteric fever. The enteric fever is exclusive to humans and is the unfortunate consequence of lack of basic amenities such as safe drinking water and proper sanitation. ${ }^{[2]}$

In a recent meta-analysis, the estimated incidence of enteric fever to be 6.9-48.4 million/year in low- and

\footnotetext{
How to cite this article: Dash D, Das P, Bhargava A, Gaikwad UN, Negi SS, Wankhede A. Estimating the burden of enteric fever in Chhattisgarh: A single-center study on culture-positive cases from a newly built tertiary care hospital. J Lab Physicians 2019;11:234-9.
} 
middle-income countries with main foci being Africa and South Asia. ${ }^{[3]}$ In the same study, South Asia fell under high incidence category for enteric fever with an incidence of $100-<500$ cases $/ 100,000$ person-years. ${ }^{[3]} \mathrm{A}$ systematic review and meta-analysis onthe prevalence of enteric fever in India estimated $9.7 \%$ and $0.9 \%$ for typhoid and paratyphoid fever, respectively, across all age groups confirmed either through culture or serology ${ }^{[4]}$ Children of school-going age group and preschool children are the most commonly affected population. ${ }^{[5-7]}$

Reports of multidrug-resistant (MDR) S. enterica serovar Typhi and Paratyphi (i.e., resistant to ampicillin, chloramphenicol, and cotrimoxazole) as well as ciprofloxacin resistance are widely published. ${ }^{[7-13]}$

Enteric fever is endemic in India. While there are many studies from the different part of the country that dealt with the prevalence and antimicrobial susceptibility trend of typhoidal salmonellae, ${ }^{[5,7,10,13-18]}$ the data are very scarce from the Central India, in general ${ }^{[19,20]}$ and no published data from the state of Chhattisgarh, in particular, a newly formed state in the Central India.

Hence, a 2-year retrospective study on blood culture-confirmed cases of typhoidal salmonellae was carried out in an attempt to determine the prevalence and pattern of antimicrobial susceptibility of typhoidal salmonellae in patients of all age group at a tertiary care hospital in the Chhattisgarh.

\section{Materials and Methods}

We conducted a retrospective analysis over a period of 2 years (December 2015-December 2017) of the blood cultures submitted to our institute in Raipur, for suspected enteric fever and associated signs and symptoms such as fever with rigors, headache, toxemia, abdominal pain, nausea, dry and coated tongue, relative bradycardia, and hepatosplenomegaly.

Blood cultures were done either with conventional method or automated blood culture system (BacT/ALERT 3D, bioMérieux, USA). The identification of the typhoidal salmonellae was done by standard biochemical reactions and serotyping (Denka Seiken, Japan). Every patient with the first positive blood culture showing growth of salmonellae was included in the analysis. Subsequent positive blood culture from the same patient was excluded from the analysis.

The antimicrobial susceptibility was done by Kirby-Bauer disc diffusion technique using ampicillin $(10 \mu \mathrm{g})$, cotrimoxazole $(1.25 / 23.75$ $\mu \mathrm{g})$, ciprofloxacin $(5 \mu \mathrm{g})$, nalidixic acid $(30 \mu \mathrm{g})$, ceftriaxone $(30 \mu \mathrm{g})$, chloramphenicol $(30 \mu \mathrm{g})$, and azithromycin $(15 \mu \mathrm{g})$ (Hi-media Laboratories, Mumbai, India) as per the Clinical and Laboratory Standards Institute (CLSI) guidelines. The CLSI interpretative criteria were used for interpretation of the result. ${ }^{[21-23]}$

The minimum inhibitory concentration (MIC) of the stored strains was also determined for ceftriaxone, ciprofloxacin, and azithromycin by E-test according to the manufacturers' instruction (HiMedia Laboratories, Mumbai, India). Interpretation of susceptibility was carried out again by the CLSI guideline. The $\mathrm{MIC}_{50}$ and $\mathrm{MIC}_{90}$ were determined by assembling the MIC values of all the strains in an "orderly array" in Microsoft Excel datasheet and then selecting the $50^{\text {th }}$ and $90^{\text {th }}$ percentile, respectively.

Escherichia coli ATCC 25922 was used as the quality control strain for antimicrobial susceptibility testing.

An isolate was considered MDR if they were resistant to ampicillin, cotrimoxazole, and chloramphenicol. ${ }^{[1]}$

We divided a year into four quarters, i.e., first quarter (January-March), second quarter (AprilJune), third quarter (July-September), and fourth quarter (October-December) to analyze whether there exists a seasonal variation or not in the occurrence of the enteric fever.

\section{Results}

A total of 1430 blood culture samples received (798 conventional and 632 automated culture bottles) at our department during this study period. Out of which, $3.56 \%$ (51 cases) were culture positive for typhoidal Salmonella, 2.5\% (36 cases) being S. enterica serovar Typhi and $1.04 \%$ (15 cases) of S. enterica serovar Paratyphi A of the total sample received. Twenty-two cases $(2.75 \%)$ out of 798 conventional culture and 29 cases $(4.58 \%)$ out of 632 automated blood culture were positive for typhoidal Salmonella. Age-wise, the highest occurrence of culture positivity was seen in the age group $10-19$ years (41.2\%) followed by $0-9$ years $(25.5 \%)$ [Table 1$]$. The median age is 14 years (range $2-43$ years), while the male: female ratio is $2: 1$.

Table 1: Age group distribution of culture-positive enteric fever $(n=51)$

\begin{tabular}{lccc}
\hline Age group & Female (\%) & Male (\%) & Grand total (\%) \\
\hline $0-9$ & 11.8 & 13.7 & 25.5 \\
$10-19$ & 15.7 & 25.5 & 41.2 \\
$20-29$ & 5.9 & 17.6 & 23.5 \\
$30-39$ & 0.0 & 7.8 & 7.8 \\
$40-49$ & 0.0 & 2.0 & 2.0 \\
Grand total & 33.3 & 66.7 & 100.0 \\
\hline
\end{tabular}


The detailed age group distribution of culture-positive enteric fever was presented in Table 1.

Fever is the most common presenting symptom being present is all the patients followed by nausea $(53 \%)$ while coated tongue is the most common presenting sign (53\%). The detailed signs and symptoms were presented in Table 2.

Among the isolated salmonellae, S. enterica serovar Typhi was 70.5\%, while S. enterica serovar Paratyphi A was $29.5 \%$.

The highest isolation (35.3\%) occurred in the third quarter (July-September) of the year followed $27.4 \%$ in the second quarter (April-June) [Figure 1].

The year-wise and quarter-wise variations in isolation of salmonellae are presented in Figures 1 and 2, respectively.

The susceptibility pattern by disc diffusion method showed $100 \%$ susceptibility to the third-cephalosporin for both S. enterica serovar Typhi and Paratyphi A. All the isolates showed resistance to ciprofloxacin when nalidixic acid was taken as a surrogate marker. However, when we determine the MIC by E strip, $95 \%$ of the isolates were of intermediate susceptibility or decreased ciprofloxacin susceptibility (DCS). One isolate of S. enterica serovar Typhi had a MIC value of $16 \mu \mathrm{g} / \mathrm{ml}$. Two isolates $(3.9 \%)$ were found to be MDR. The susceptibility pattern of $S$. enterica serovar Typhi and S. enterica serovar Paratyphi A is shown in Table 3.

The $\mathrm{MIC}_{50}$ and $\mathrm{MIC}_{90}$ of ciprofloxacin was found to be $0.125 \mu \mathrm{g} / \mathrm{ml}$ and $6.514 \mu \mathrm{g} / \mathrm{ml}$, respectively, for S. enterica serovar Typhi (mean $1.2 \mu \mathrm{g} / \mathrm{ml}$ ) while that for S. enterica serovar Paratyphi A, it was $0.38 \mu \mathrm{g} / \mathrm{ml}$ and $16 \mu \mathrm{g} / \mathrm{ml}$, respectively (mean $1.97 \mu \mathrm{g} / \mathrm{ml}$ ).
All the isolates were susceptible to ceftriaxone, and the mean, $\mathrm{MIC}_{50}$ and $\mathrm{MIC}_{90}$ for S. enterica serovar Typhi $0.098 \mu \mathrm{g} / \mathrm{ml}, 0.094 \mu \mathrm{g} / \mathrm{ml}$, and $0.125 \mu \mathrm{g} / \mathrm{ml}$, respectively, while that for S. enterica serovar Paratyphi A, it is $0.143 \mu \mathrm{g} / \mathrm{ml}, 0.125 \mu \mathrm{g} / \mathrm{ml}$ and $0.19 \mu \mathrm{g} / \mathrm{ml}$, respectively.

$\mathrm{The}_{\mathrm{MIC}}$ value for azithromycin in S. enterica serovar Typhi and S. enterica serovar Paratyphi A was 8 and $12 \mu \mathrm{g} / \mathrm{ml}$, respectively, while $\mathrm{MIC}_{90}$ was 16 and $19.2 \mu \mathrm{g} / \mathrm{ml}$.

\section{Discussion}

The enteric fever continues to be a major public health menace in developing countries. It also attracts significant attention among travelers due to increased global mobility. The predominant serotypes in India as well as in neighboring countries are S. enterica serovar Typhi and S. enterica serovar Paratyphi A. ${ }^{[6,9,24]}$ There is also an increased report of antimicrobial resistance to once susceptible drugs globally. Hence, there is a need for constant vigilance on the disease burden as well as on the changing pattern of the antimicrobial susceptibility.

Chhattisgarh is a relatively newly formed state in the Central India with coordinates $21.278^{\circ} \mathrm{N}, 81.866^{\circ}$ E. Ours'

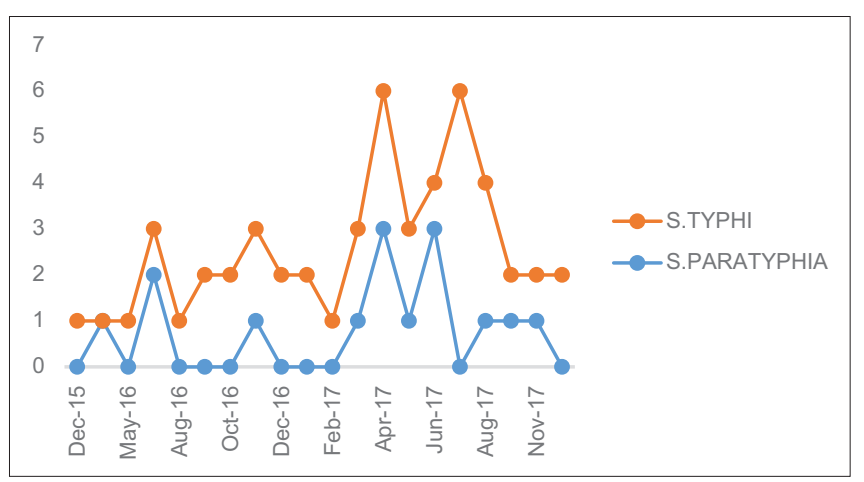

Figure 1: Year-wise distribution of enteric fever

Table 2: Symptoms and signs of the culture-positive enteric fever $(n=51)$

\begin{tabular}{lclc}
\hline Symptoms & Percentage of patients presented with & Signs & Percentage of patients presented with \\
\hline Fever & 100 & Toxemia & 49 \\
Nausea & 53 & Coated tongue & 53 \\
Abdominal pain & 41 & Hepatosplenomegaly & 23 \\
Headache & 42 & & \\
\hline
\end{tabular}

Table 3: Susceptibility pattern of S. enterica serovar Typhi and S. enterica serovar Paratyphi A

\begin{tabular}{lcccc}
\hline Drugs & \multicolumn{2}{c}{ Susceptibility pattern for Salmonella typhi $(n=36)$} & Susceptibility pattern for Salmonella f para Typhi A $(n=15)$ \\
\cline { 2 - 5 } & Resistant (\%) & Susceptible $(\%)$ & Resistant $(\%)$ & Susceptible $(\%)$ \\
\hline Ampicillin & 16.7 & 83.3 & 6.7 & 93.3 \\
Chloramphenicol & 8.3 & 91.7 & 0.0 & 100.0 \\
Cotrimoxazole & 8.3 & 91.7 & 6.7 & 93.3 \\
Ciprofloxacin & 100.0 & 0.0 & 100.0 & 0.0 \\
Ceftriaxone & 0.0 & 100.0 & 0.0 & 100.0 \\
\hline
\end{tabular}


is a newly established Government Medical College and Research Institute funded by the Government of India and situated in Raipur. In the present study, we analyzed a 2-year data from December 2015 (i.e., since we started receiving blood samples for culture formally) to December 2017 of blood culture received with a provisional diagnosis of enteric fever and associated symptoms retrospectively.

In the present study, 51 (3.56\%) blood cultures were positive for typhoidal Salmonella (S. serovars Typhi 2.5\% and Paratyphi A 1.04\%). In a recent 10 years retrospective study by Sharma et al. at All India Institute of Medical Sciences, New Delhi, found the overall culture positive rate of $0.32 \%$, S. enterica serovars Typhi and Paratyphi A being $0.23 \%$ and $0.09 \%$, respectively. ${ }^{[7]}$ Similarly, Iyer et al. from Hyderabad found the isolation rate of $S$. enterica serovars Typhi and Paratyphi A in children were $0.5 \%$ and $0.1 \%$, respectively, in a 10 -years retrospective study. ${ }^{[5]}$ The World Health Organization conducted study in five Asian countries (China, India, Indonesia, Pakistan, and Vietnam) among children in 2008 found a culture positivity rate of $2 \% .^{[6]}$ The relatively high isolation rate in our study may be due to a high endemic zone of enteric fever which has never been reported or the patient was not exposed to inadvertent antimicrobials use due to poor access to health-care facilities.

For a targeted intervention approach, an awareness ofthe age-specific prevalence of enteric fever is of paramount importance. In our study, though the enteric fever-affected patients across all the age groups, the highest isolation was seen in children of $10-19$ years $(41.2 \%)$ followed by children of 0-9 years (25.5\%) [Table 1], the median age is 14 years. Similar results were presented in other studies also. ${ }^{[5-7]}$ Time and again, this finding underscores the idea of full evaluation for enteric fever in any children and adolescent with febrile illness, especially in endemic areas.

Enteric fever may present with varied sign and symptoms. Fever is the most common presenting

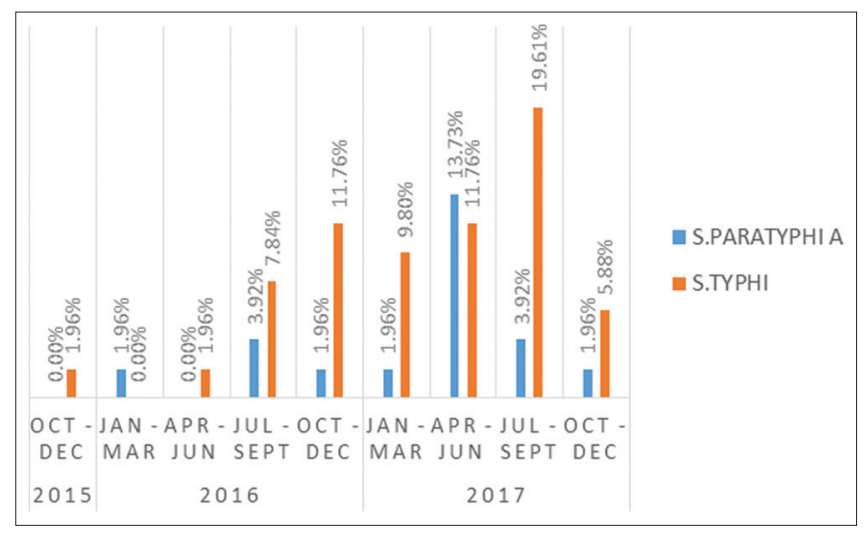

Figure 2: Quarter-wise distribution of culture-positive cases of enteric fever symptom in this current study, while coated tongue is the most common presenting sign. Sinha et al. also found similar result in children under 5 years of age. ${ }^{[25]}$ Toxic look and coated tongue were also the common presenting signs in other studies. ${ }^{[26,27]}$

Among the isolated salmonellae in this study, S. enterica serovar Typhi was the predominant isolate $(70.5 \%)$ followed by S. enterica serovar Paratyphi A (29.5\%). Neither S. enterica serovar Paratyphi B, Paratyphi C, nor any nontyphoidal Salmonella was isolated. Other Indian studies also have reported similar findings. ${ }^{[5,7,8,14]}$ However, there are also reports of increased incidence of S. enterica serovar Paratyphi A infections ${ }^{[15,28]}$ and also a shifting of trend from S. enterica serovar Typhi to $S$. enterica serovar Paratyphi A. [4,7,29-31] $^{2}$

The enteric fever though occurs throughout the year in the endemic area, and it does follow a seasonal pattern with increase of cases with increase in rainfall. ${ }^{[32]}$ In the current study also, we found similar seasonal variation, isolation rate being highest in the July-September (35.3\%) in the monsoon season followed by April-June (27.4\%) in the summer and premonsoon season. Similar results were also seen elsewhere. ${ }^{[5,24,33]}$ It has been suggested that with increase in rainfall, there is chance of fecal contamination of surface water which may lead to increased incidence of enteric fever. ${ }^{[5,24,33,34]}$ Typhoid fever spread predominantly within the household apparently by convalescent cases transiently excreting the bacterium, while the paratyphoid fever largely follows an extra household transmission. ${ }^{[31,33,34]}$ While we do believe that the increased incidence of enteric fever in July-September in our study is also due to fecal contamination of surface water during the monsoon season, we attribute the increase in incidence in AprilJune is due to increased consumption of flavored ice candies and cold beverages from roadside vendors, with the onset of summer in this part of the country. However, this attribution is purely conjectural and further evaluation needs to be carried out to establish a causal relationship.

There are reports of gradual decrease in the prevalence of enteric fever. ${ }^{[4,7]}$ In our study, there is an increased case of enteric fever in 2017 than 2016 [Figure 1]. This may be due to increased patient load in this newly established institute and also shifting of blood culture method from conventional to automation as our study showed $4.58 \%$ culture positivity rate as compared to $2.75 \%$ in conventional methods. To know the exact trend in this area, further follow-up is necessary.

Timely diagnosis and appropriate antimicrobial treatment of enteric fever is the key to successful management and to reduce the complications. It also 
helps in prevention of carrier state in enteric fever. In the present study, ciprofloxacin found to be $100 \%$ resistant when nalidixic acid disc diffusion criteria were taken as a surrogate marker for ciprofloxacin susceptibility, but in MIC determination by E-strip showed $95 \%$ of Salmonella were DCS to ciprofloxacin, i.e., they have a MIC range in between 0.125 and $0.5 \mu \mathrm{g} / \mathrm{ml}$. The $\mathrm{MIC}_{50}$ of S. enterica serovar Typhi and Paratyphi A were 0.125 and $0.38 \mu \mathrm{g} / \mathrm{ml}$, respectively, while the $\mathrm{MIC}_{90}$ were 6.514 and $16.0 \mu \mathrm{g} / \mathrm{ml}$ for the two serovars in that order. Since CLSI revised the breakpoints for Salmonella from $1 \mu \mathrm{g} /$ $\mathrm{ml}$ to $0.06 \mu \mathrm{g} / \mathrm{ml}$ in 2012, there are reports of increased resistance to ciprofloxacin. ${ }^{[5,7,9]}$ This is due to widespread prescription of fluoroquinolones as oral preparations are available, can be bought over the counter and affordable. ${ }^{[10]}$ The decreased susceptibility is reached to such point that it is no longer a drug of choice for enteric fever. ${ }^{[1]}$

At present, the third-generation cephalosporin such as ceftriaxone, cefixime, and azithromycin, a macrolide are drug of choice for treatment of enteric fever. In our study, there is $100 \%$ susceptibility to ceftriaxone with $\mathrm{MIC}_{50}$ for S. enterica serovar Typhi and Paratyphi A were 0.094 and $0.125 \mu \mathrm{g} / \mathrm{ml}$, respectively. But now, ceftriaxone MIC creeping toward resistance has been reported by Sharma et al. ${ }^{[7]}$

Azithromycin is an orally available macrolide. While all the S. enterica serovar Typhi were susceptible to azithromycin, the susceptibility for $S$. enterica serovar Paratyphi A cannot be commented upon as presently there is no defined guidelines in the CLSI. Here, it can be inferred that the overall MIC distribution in S. enterica serovar Paratyphi A was higher in comparison to S. enterica serovar Typhi across all the antimicrobials. In the present study, all the isolates were susceptible to azithromycin with $\mathrm{MIC}_{50}$ for $S$. enterica serovar Typhi and Paratyphi A were 8.0 and $12.0 \mu \mathrm{g} / \mathrm{ml}$, respectively. Similar results were also seen in a study by Iyer et al. ${ }^{[5]}$ There are reports of azithromycin resistance and treatment failure. ${ }^{[35,36]}$ Fear have been expressed that azithromycin may meet the same fate as that of ciprofloxacin in management of enteric fever, as this is a common drug to be prescribed in respiratory tract infections in India, has oral preparation available, once a day dosing and over-the-counter availability. ${ }^{[2]}$

The susceptibility toward the first-line drugs such as ampicillin, chloramphenicol, and cotrimoxazole are $83.3 \%, 91.7 \%$, and $91.7 \%$, respectively, for S. enterica serovar Typhi. Only two isolates $(3.9 \%)$ of S. enterica serovar Typhi and none of S. enterica serovar Paratyphi A were MDR (resistant to ampicillin, chloramphenicol, and cotrimoxazole) in the present study. A declining trend of MDR typhoidal salmonellae has been reported all over the country. ${ }^{[5,8,10,14]}$ This may be due to near negligent prescription of these first-line drugs in the present time. These drugs should not be used empirically for enteric fever and only prescribed after availability of antimicrobial susceptibility pattern of each individual isolate.

Our study has limitations. The study duration is short to actually comment on the trend of enteric fever in this region. The cases presented to our institute might be the cases of treatment failure and might not represent the true prevalence of the disease in the community. The $\mathrm{MIC}_{50}$ and $\mathrm{MIC}_{90}$ were determined from a small population of organism which may not have represented the true MIC values. This was an entirely laboratory-based study on culture positive enteric fever where consideration of the actual therapeutic intervention and clinical outcome of the patients were not taken into account.

\section{Conclusion}

This study reflects the prevalence and antimicrobial susceptibility pattern of typhoidal Salmonella in this region. There is a high prevalence of the disease which needs urgent focus on safe water, sanitation services, and community education by the public health department. The enteric fever affects persons across all the age group with a greater isolation rate in children calling for a targeted intervention in school-going children. The disease is present throughout the year with a seasonal peak in monsoon followed by onset of summer. S. typhi is the most common serovar isolated. A very high resistance to ciprofloxacin is indicated by this study. A $100 \%$ susceptibility to ceftriaxone and azithromycin makes them a rational choice for the first-line therapy. Although the percentage of MDR typhoidal salmonellae is very low as seen in this study, ampicillin, chloramphenicol, and cotrimoxazole, the once first-line drugs for enteric fever should be used cautiously and only after obtaining the antibiotic susceptibility report of individual patient.

\section{Financial support and sponsorship \\ Nil.}

\section{Conflicts of interest}

There are no conflicts of interest.

\section{References}

1. Crump JA, Sjölund-Karlsson M, Gordon MA, Parry CM. Epidemiology, clinical presentation, laboratory diagnosis, antimicrobial resistance, and antimicrobial management of invasive salmonella infections. Clin Microbiol Rev 2015;28:901-37.

2. Veeraraghavan B, Pragasam AK, Bakthavatchalam YD, Ralph R. Typhoid fever: Issues in laboratory detection, treatment options concerns in management in developing countries. Future Sci OA 2018;4:FSO312. 
3. Antillón M, Warren JL, Crawford FW, Weinberger DM, Kürüm E, Pak GD, et al. The burden of typhoid fever in low- and middle-income countries: A meta-regression approach. PLoS Negl Trop Dis 2017;11:e0005376.

4. John J, Van Aart CJ, Grassly NC. The burden of typhoid and paratyphoid in India: Systematic review and meta-analysis. PLoS Negl Trop Dis 2016;10:e0004616.

5. Iyer RN, Jangam RR, Jacinth A, Venkatalakshmi A, Nahdi FB. Prevalence and trends in the antimicrobial susceptibility pattern of Salmonella enterica serovars typhi and paratyphi A among children in a pediatric tertiary care hospital in South India over a period of ten years: A retrospective study. Eur J Clin Microbiol Infect Dis 2017;36:2399-404.

6. Ochiai RL, Acosta CJ, Danovaro-Holliday MC, Baiqing D, Bhattacharya SK, Agtini MD, et al. A study of typhoid fever in five Asian countries: Disease burden and implications for controls. Bull World Health Organ 2008;86:260-8.

7. Sharma P, Dahiya S, Manral N, Kumari B, Kumar S, Pandey S, et al. Changing trends of culture-positive typhoid fever and antimicrobial susceptibility in a tertiary care North Indian hospital over the last decade. Indian J Med Microbiol 2018;36:70-6.

8. Joshi S. Antibiogram of S. enterica serovar typhi and S. enterica serovar paratyphi A: A multi-centre study from India. WHO South East Asia J Public Health 2012;1:182-8.

9. Akhtar S, Sarker MR, Jabeen K, Sattar A, Qamar A, Fasih N. Antimicrobial resistance in Salmonella enterica serovar typhi and paratyphi in South Asia-current status, issues and prospects. Crit Rev Microbiol 2015;41:536-45.

10. Capoor MR, Nair D. Quinolone and cephalosporin resistance in enteric fever. J Glob Infect Dis 2010;2:258-62.

11. Sharma P, Dahiya S, Kumari B, Balaji V, Sood S, Das BK, et al. Pefloxacin as a surrogate marker for quinolone susceptibility in Salmonella enterica serovars typhi and paratyphi A in India. Indian J Med Res 2017;145:687-92.

12. Chaudhari DM, Renjen PN, Sardana R, Butta H. Nocardia farcinica brain abscess in an immunocompetent old patient: A case report and review of literature. Ann Indian Acad Neurol 2017;20:399-402.

13. Menezes GA, Harish BN, Khan MA, Goessens W, Hays JP. Antimicrobial resistance trends in blood culture positive Salmonella paratyphi A isolates from Pondicherry, India. Indian J Med Microbiol 2016;34:222-7.

14. Choudhary A, Gopalakrishnan R, Nambi PS, Ramasubramanian V, Ghafur KA, Thirunarayan MA. Antimicrobial susceptibility of Salmonella enterica serovars in a tertiary care hospital in Southern India. Indian J Med Res 2013;137:800-2.

15. Palit A, GhoshS, DuttaS, Sur D, Bhattacharya MK, Bhattacharya SK. Increasing prevalence of Salmonella enterica serotype paratyphi-A in patients with enteric fever in a Periurban slum setting of Kolkata, India. Int J Environ Health Res 2006;16:455-9.

16. Basu S, Dewan ML, Suri JC. Prevalence of salmonella serotypes in India: A 61-year study. Bull World Health Organ 1975;52:331-6.

17. Gautam V, Gupta NK, Chaudhary U, Arora DR. Sensitivity pattern of Salmonella serotypes in Northern India. Braz J Infect Dis 2002;6:281-7.

18. Kanungo S, Dutta S, Sur D. Epidemiology of typhoid and paratyphoid fever in India. J Infect Dev Ctries 2008;2:454-60.

19. Chande C, Shrikhande S, Kapale S, Agrawal S, Fule RP. Change in antimicrobial resistance pattern of Salmonella typhi in central India. Indian J Med Res 2002;115:248-50.
20. Chitnis S, Chitnis V, Hemvani N, Chitnis DS. Ciprofloxacin therapy for typhoid fever needs reconsideration. J Infect Chemother 2006;12:402-4.

21. Clinical and Laboratory Standards Institute. M 100- S26 Performance Standards for Antimicrobial Susceptibility Testing; Twenty-Sixth Informational Supplement. Wayne, Pa: Clinical and Laboratory Standards Institute; 2016.

22. Clinical and Laboratory Standards Institute. M -100 S25 Performance Standards for Antimicrobial Susceptibility Testing: Twenty-Fifth Informational Supplement. Wayne, PA: Clinical and Laboratory Standards Institute; 2015.

23. Clinical and Laboratory Standards Institute. M 100 S27 Performance Standards for Antimicrobial Susceptibility Testing: Twenty-Seventh Informational Supplement. Wayne, PA: Clinical and Laboratory Standards Institute; 2017.

24. Dewan AM, Corner R, Hashizume M, Ongee ET. Typhoid fever and its association with environmental factors in the Dhaka metropolitan area of Bangladesh: A spatial and time-series approach. PLoS Negl Trop Dis 2013;7:e1998.

25. Sinha A, Sazawal S, Kumar R, Sood S, Reddaiah VP, Singh B, et al. Typhoid fever in children aged less than 5 years. Lancet 1999;354:734-7.

26. Modi R. Clinical profile and treatment outcome of typhoid fever in children at a teaching hospital, Ahmedabad, Gujarat, India. Int J Med Sci Public Health 2016;5:212.

27. Devaranavadagi RA, Srinivasa S. A study on clinical profile of typhoid fever in children. Int J Contemp Pediatr 2017;4:1067-73.

28. Bhattacharya SS, Dash U. A sudden rise in occurrence of Salmonella paratyphi a infection in Rourkela Orissa. Indian J Med Microbiol 2007;25:78-9.

29. Sood S, Kapil A, Dash N, Das BK, Goel V, Seth P. Paratyphoid fever in India: An emerging problem. Emerg Infect Dis 1999;5:483-4.

30. Teh CS, Chua KH, Thong KL. Paratyphoid fever: Splicing the global analyses. Int J Med Sci 2014;11:732-41.

31. Bharmoria A, Vaish VB, Tahlan AK, Majumder S. Analysis of attributing characteristics of Salmonella enterica serovar Paratyphi A, B and C across India during 6 years (2010 to 2015). Med Microbiol Diagn 2016;5:220-4. Available from: https:// www.omicsonline.org/open-access/analysis-of-attributingcharacteristics-of-salmonella-enterica-serovarparatyphi-a-b-andc-across-india-during-6-years-2010-to-2015-2161-0703-1000220. php?aid=69878. [Last accessed on 2018 Jun 10].

32. Saad NJ, Lynch VD, Antillón M, Yang C, Crump JA, Pitzer VE. Seasonal dynamics of typhoid and paratyphoid fever. Sci Rep 2018;8:6870.

33. Vollaard AM, AliS, van Asten HA, Widjaja S, Visser LG, Surjadi C, et al. Risk factors for typhoid and paratyphoid fever in Jakarta, Indonesia. JAMA 2004;291:2607-15.

34. Black RE, Cisneros L, Levine MM, Banfi A, Lobos H, Rodriguez H. Case-control study to identify risk factors for paediatric endemic typhoid fever in Santiago, Chile. Bull World Health Organ 1985;63:899-904.

35. Rai S, Jain S, Prasad KN, Ghoshal U, Dhole TN. Rationale of azithromycin prescribing practices for enteric fever in India. Indian J Med Microbiol 2012;30:30-3.

36. Manesh A, Balaji V, Kumar DR, Rupali P. A case of clinical and microbiological failure of azithromycin therapy in Salmonella enterica serotype typhi despite low azithromycin MIC. Int J Infect Dis 2017;54:62-3. 